included; decline in renal function during pregnancy (defined as $25 \%$ increase in serum creatinine), persistent decline in renal function six months post-partum and time to renal replacement therapy. Results 49 women (57 pregnancies) with CKD stages 3-5 were identified with sufficient data for analysis. Diabetes mellitus was the underlying diagnosis in nine (16\%) pregnancies. 18 (32\%) pregnancies were complicated by chronic hypertension. 11 (19\%) pregnancies occurred in women with previous renal transplant. $21 \%$ of women had $>1 \mathrm{~g} /$ day proteinuria prior to pregnancy.

$11(22 \%)$ women had a decline in renal function in pregnancy. Decline in renal function at 6 months post-partum was present in a total of $16(33 \%)$ women. Predictors of significant decline in renal function included; pre-pregnancy creatinine mean $222 \mu \mathrm{mol} / \mathrm{L}$ in those with decline in function and $138 \mu \mathrm{mol} / \mathrm{L}$ in those without $(p=0.0015)$, and significant proteinuria $>1 \mathrm{~g} /$ day present in $87.5 \%(p=0.02)$.

Nine $(18 \%)$ women went on to need renal replacement therapy (RRT), four within a year of delivery. Mean time to RRT was 23 months. Conclusions Pregnant women with CKD 3-5 are at high risk of deterioration in renal function during and after pregnancy. Level of creatinine prior to pregnancy and significant proteinuria are risk factors for decline in function.

\section{PM.99 SYPHILIS SEROLOGY IN PREGNANT WOMEN OVER A PERIOD OF 7 YEARS (2005-2011) IN A LARGE MATERNITY HOSPITAL IN DUBLIN, IRELAND}

doi:10.1136/archdischild-2013-303966.180

'A Varughese, 'V Jackson, 'M Cafferkey, 'M Brennan, 'M Lawless, 'V Ciprike, 'M Eogan, 'W Ferguson, 'S Coulter-Smith, 1.2J Lambert. 'The Rotunda Hospital, Dublin, Ireland; ${ }^{2}$ The Mater Misericordiae University Hospital, Dublin, Ireland

Nearly 1.5 million pregnant women are infected with probable active syphilis each year, and approximately half of infected pregnant women who are untreated, will experience adverse outcomes due to syphilis, such as early fetal loss and stillbirth, neonatal death, lowbirth-weight infants, and infants with clinical evidence of infection.

Data for all patients with positive treponemal serology at booking visit from 2005 to 2011 was gathered.

Between 2005 and 2011, 179/64349 women had positive syphilis serology representing $0.28 \%$ of the patient population. These women were between the age of 19 and 41 with a higher prevalence among women of East European origin. In the 7 year period, 1 case of congenital syphilis was recorded. This patient was a DCDA twin pregnancy who booked late at 22 weeks and delivered prematurely at 23 weeks. Hence, syphilis treatment was not commenced.

This study highlights the continued prevalence of positive syphilis serology in our pregnant population. Our combined obstetric and infectious disease clinic optimises opportunities for appropriate treatment and follow-up. Contact tracing and screening for other sexually transmitted infections are also vital components of this service.

In the current economic climate, with continued emphasis on provision of cost-effective healthcare it is important to justify the cost of screening 67921 women to identify 179 cases. Untreated syphilis has a range of antenatal and paediatricsequelae and thus we recommend that screening for syphilis continues, particularly considering frequent migration of women from Eastern Europe to this country.

\section{PM.100 A RARE CASE OF ANTI-NMDA RECEPTOR ENCEPHALITIS IN PREGNANCY}

doi:10.1136/archdischild-2013-303966.181

${ }^{1,}{ }^{\mathrm{E}} \mathrm{E}$ Gaughan, ${ }^{2} \mathrm{~S}$ Barry, ${ }^{2} \mathrm{~A}$ McHugh, ${ }^{2} \mathrm{~F}$ O'Donoghue, ${ }^{2} \mathrm{~K}$ O'Rourke, ${ }^{2} \mathrm{~T}$ Lynch, ${ }^{2,3} \mathrm{~A}$ McCarthy, ${ }^{1,2} \mathrm{~J}$ Sheehan, ${ }^{1,2 \mathrm{P}}$ McKenna. 'Rotunda Hospital, Dublin, Ireland; ' ${ }^{2}$ Mater Hospital, Dublin, Ireland; ${ }^{3}$ Holles St, Dublin, Ireland

Anti-NMDA receptor encephalitis is a distinct disorder characterised by the predictable sequential development of symptoms; prodromal symptoms are initially noted, followed by prominent psychiatric symptoms, seizures, an unresponsive/catatonic state, hypoventilation, and involuntary orofacial-limb movements. This disorder usually affects young women with ovarian teratoma but may also affect women of any age or even men.

We report the case of a 32 year old primigravid woman who developed psychosis with associated catatonia and autonomic dysfunction at 8 weeks gestation. Cranial imaging in the form of CT and MRI was normal. EEG showed slow waves and anti-NMDA receptor encephalitis was suspected. This was confirmed by the finding of serum anti-NMDA antibodies. Transvaginal Ultrasound and pelvic MRI suggested normal ovaries. She required admission to the High Dependency Unit for several weeks but eventually responded to plasma exchange, steroids, azathioprine, Intravenous immunoglobulin and antipsychotics.

She had an Emergency LSCS at 32 weeks gestation for PPROM and delivered a healthy male infant. A mature cystic teratoma was found at caesarean section which was excised.

Although being rare in pregnancy, anti NMDA encephalitis can respond to aggressive treatment and can be associated with good maternal and fetal outcomes. An awareness of this 'new disease' (first described in 2005) can lead to an occasional but dramatic surgical treatment of a psychotic illness.

\section{PM.101 LOCALLY INVASIVE OESOPHAGEAL ADENOCARCINOMA DIAGNOSED IN THE SECOND TRIMESTER, THE OPTIONS}

doi:10.1136/archdischild-2013-303966.182

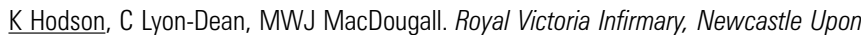
Tyne, UK

Oesophageal cancer is a rare malignancy to present during pregnancy. Standard treatment outside of pregnancy involves three cycles of Cisplatin, Epirubacin and 5 Flurouracil, a combination rarely used in pregnancy, followed by oesophagectomy in week 15 of treatment. We aim to highlight the management dilemmas posed by such cases, balancing the risk of treatment options in trying to achieve the best outcome for both mother and baby.

We present the case of a 31 year old primiparous woman diagnosed at 23 weeks gestation with locally invasive oesophageal adenocarcinoma.

Delay in chemotherapy treatment with early delivery by Caesarean section risked disease progression to an inoperable stage for mum, and the risk of prematurity for baby.

We elected to start three cycles of the standard chemotherapy regime without delay, with increased fetal surveillance. Maternal anaemia developed. Prostaglandin induction took place at $37+2$ weeks gestation, resulting in a forceps delivery of a live $2050 \mathrm{~g}$ male infant with Apgars of 9 and 9 at one and 5 minutes. Neonatal full blood count was normal at 12 hours of age. Postnatal staging confirmed the lesion was still operable and oesophagectomy took place at the usual chemotherapy surgery time interval. Good tumour margins were achieved and the patient returned home on day ten.

We discuss our rational not to delay treatment with early delivery by Caesarean section, accepting the risk of chemotherapy in pregnancy. We review the literature surrounding this cocktail of chemotherapeutic agents and discuss the dilemmas surrounding the treatment of her anaemia.

\section{PM.102 THE EFFECT OF INTRODUCING A VENOUS THROMBOEMBOLISM RISK ASSESSMENT TOOL ON THE WORKLOAD OF A COMBINED OBSTETRIC-HAEMATOLOGY CLINIC}

doi:10.1136/archdischild-2013-303966.183

${ }^{1} \mathrm{G}$ Wright, ' $\mathrm{J}$ Moore, ' $\mathrm{G}$ Swallow, ${ }^{2} \mathrm{~B}$ Myers. 'Nottingham Univeristy Hospitals NHS Trust, City Hospital Campus, Nottingham, UK; '2 Lincoln County Hospital, Lincoln, UK 